\title{
On Spectral Approximations of Unbounded Operators
}

\author{
Marian Dmytryshyn ${ }^{1} \cdot$ Oleh Lopushansky $^{2}$ (1) \\ Received: 19 November 2017 / Accepted: 19 April 2019 / Published online: 2 May 2019 \\ (c) The Author(s) 2019
}

\begin{abstract}
We establish an improvement of Bernstein-Jackson inequalities by explicitly calculating constants on special approximation scales of analytic vectors of finite exponential types, generated by unbounded operators. Inequalities are applied to analytical estimates of spectral approximations of unbounded operators. Applications to spectral approximations of elliptic and ordinary differential boundary-value problems are shown.
\end{abstract}

Keywords Spectral approximations · Bernstein-Jackson inequalities

Mathematics Subject Classification 47A58 · 41A17

\section{Introduction}

We investigate a spectral approximation problem for a linear closed unbounded operator $A$ in a Banach space $X$, using the subspace $\mathscr{E}(A) \subset X$ of its analytic vectors of finite exponential types. We call this the spectral approximation because in the case of operators $A$ with discrete spectrum the subspaces $\mathscr{E}(A)$ exactly coincide with the linear span of all its spectral subspaces $\mathscr{R}(A)$ [5]. For many other operators (see e.g., [13]), the subspace $\mathscr{E}(A)$ also contains all their spectral subspaces. Non-triviality of $\mathscr{E}(A)$, for example, in the case of generators $A$ of strongly continuous 1-parameter groups, is checked in Proposition 1.

Communicated by Jussi Behrndt, Fabrizio Colombo, Sergey Naboko.

$凶$ Oleh Lopushansky

ovlopusz@ur.edu.pl

Marian Dmytryshyn

marian.dmytryshyn@pnu.edu.ua

1 Precarpathian National University, 57 Shevchenka str., Ivano-Frankivsk 76-018, Ukraine

2 Rzeszów University, 1 Pigonia str., 35-310 Rzeszow, Poland

Birkhäuser 
The basic tool in our approach is the functional $E(t, x ; \mathscr{E}(A), X)$ (more details in $[2,16])$ which in our cases characterizes the shortest distance from $x \in X$ to a subspace of vectors with an exponential type not larger than $t>0$. We use an adaptation of approximation scales (in terms of [17]) of quasi-normed Besov spaces $\mathscr{B}_{\tau}^{S}(A)$, determined by $E(t, x ; \mathscr{E}(A), X)$, which are connected with the approximation errors by Bernstein-Jackson type inequalities.

Earlier applications of analytic vectors of finite exponential types to approximation problems can be found in $[7,8]$. Looking historically, the idea of exponential type vectors comes from analytic vectors in Nelson's theorem [14]. Analyses of various estimates via approximation functionals are carried out in [1] and in other publications. In $[9,10]$ instead of the functional $E(t, x ; \mathscr{E}(A), X)$, a modulus of smoothness $\omega(\cdot)$ was used.

One of our aims is to prove the inverse and direct theorems that give an estimate of approximation errors by means of elements $\mathscr{E}(A)$. Namely, the inverse Theorem 2(a) which is usually identified with Bernstein's inequality (1) and the direct Theorem 2(b) which is identified with Jackson's inequality (2). Here, the main result is that in the inequalities (1)- (2) we obtain the explicit dependence of constants $c_{s, \tau}, C_{s, \tau}$ on parameters of the Besov spaces $\mathscr{B}_{\tau}^{s}(A)$. The calculated constants are exact in the sense that the limits (3) of direct and inverse sequences as $\tau \rightarrow \infty$ coincide with 1 . These theorems characterize subclasses of elements from $X$ in relation to rapidity of approximations. In our case, these subclasses are completely described by the quasi-normed Besov-type spaces $\mathscr{B}_{\tau}^{S}(A)$.

Theorem 2 uses the completeness of quasi-normed space $\mathscr{E}(A)$, which previously is proved in Theorem 1(a). The proof of completeness is based on Bernstein's compactness principle for entire analytic functions of exponential type. Under an implicit assumption of this completeness, the second part of this theorem in a somewhat different form was given in [6, Thm 3(i)].

It is important that in the case, when $A$ is the operator of differentiation $D$ in $L_{p}(\mathbb{R})$, the scale of Besov spaces $\mathscr{B}_{\tau}^{S}(A)$, as well as, the Bernstein-Jackson inequalities fully coincide with known classical analogs (see [6, Thm 7-8]). However, in this case, the exact values of constants in these inequalities were not earlier calculated.

The last two sections are applications. It is essential that estimates of spectral approximations are given in terms of quasi-norms of classic Besov spaces what are well investigated.

If $A$ is a regular elliptic operator with variable smooth coefficients over the space $L_{p}(\Omega)$ on a bounded domain $\Omega \subset \mathbb{R}^{n}$, we prove in Theorem 3 that the space $\mathscr{B}_{\tau}^{s}(A)$ coincides with an appropriate subspace in the classic Besov space $B_{p, \tau}^{s}(\Omega)$ and the Bernstein-Jackson inequalities for the last space give estimates of spectral approximations. A partial case of constant coefficients in elliptic boundary-value problems was considered earlier in [6].

Second example of analytic estimates of spectral approximations errors for some self-adjoint ordinary differential boundary-value problems is described in Theorem 4. 


\section{Approximation Scales Generated by Unbounded Operators}

In what follows, let $A: \mathfrak{D}(A) \rightarrow X$ be a closed linear operator with dense domain $\mathfrak{D}(A)$ in a complex Banach space $(X,\|\cdot\|)$. Set $\mathfrak{D}^{k+1}(A):=\left\{x \in \mathfrak{D}^{k}(A): A^{k} x \in \mathfrak{D}(A)\right\}$ and $\mathfrak{D}^{\infty}(A):=\bigcap_{k \in \mathbb{N}} \mathfrak{D}^{k}(A)$ where all integer powers $A^{k}$ are assumed closed. We associate with an element $x \in \mathfrak{D}^{\infty}(A)$ the scalar functions

$$
x(z)=\sum_{k=0}^{\infty} \frac{\left\|A^{k} x\right\|}{k !} z^{k} \text { and } \hat{x}(z)=\sum_{k=0}^{\infty} \frac{\left\|A^{k} x\right\|}{z^{k+1}}
$$

in variable $z \in \mathbb{C}$, interconnected by Laplace's transform.

An element $x \in \mathfrak{D}^{\infty}(A)$ is called the vector of exponential type $v>0$ of $A$, if one of the following equivalent conditions holds (see, e.g. [3, Thm 1.1.1]):

(i) $x(z)$ is entire with the exponential type $v=\lim _{\sup } \rightarrow \infty \frac{\ln M(r)}{r}$ with $M(r)=$ $\max _{|\lambda|=r}|x(\lambda)|$

(ii) the power series $\hat{x}(z)$ is such that $v=\lim _{\sup _{k \rightarrow \infty}}\left\|A^{k} x\right\|^{1 / k}<\infty$.

We consider the subspace of all exponential type vectors $\mathscr{E}(A)$ as the union $\bigcup_{v>0} \mathscr{E}^{v}(A)$ which is endowed with the quasinorm

$$
|x|_{\mathscr{E}(A)}=\|x\|+\inf \left\{v>0: x \in \mathscr{E}^{v}(A)\right\},
$$

where for any $v>0$ the subspace $\mathscr{E}^{v}(A)=\{x \in \mathscr{E}(A): \hat{x}(v)<\infty\}$ is endowed with norm

$$
\|x\|_{v}:=v \hat{x}(v)
$$

Then the continuous embedding $\mathscr{E}^{\vee}(A) \rightarrow \mathscr{E}^{\mu}(A)$ with $\mu>v$ holds and each $\mathscr{E}^{v}(A)$ is $A$-invariant, as well as, the restriction $\left.A\right|_{\mathscr{E}^{v}}(A)$ is a bounded operator (see [6, Thm 1]).

We assume that exponential type vectors $\mathscr{E}(A)$ are dense in $X$. Such an assumption is not restrictive, since $\mathscr{E}(A)$ contains spectral subspaces of $A$ (see, e.g., Propositions 1 and Remark 3 or [5, Thm 2.2]).

To investigate approximation errors, we consider a special scale of Besov-type spaces $\mathscr{B}_{\tau}^{S}(A)=\left\{x \in X:|x|_{\mathscr{B}_{\tau}^{s}(A)}<\infty\right\}$ with a pair indexes $\{0<s<\infty, 0<\tau \leq \infty\}$ or $\{0 \leq s<\infty, \tau=\infty\}$, where

$$
|x|_{\mathscr{B}_{\tau}^{s}(A)}=\left\{\begin{array}{lr}
\left(\int_{0}^{\infty}\left[t^{s} E(t, x ; \mathscr{E}(A), X)\right]^{\tau} \frac{d t}{t}\right)^{1 / \tau}, & 0<\tau<\infty, \\
\sup _{t>0} t^{s} E(t, x ; \mathscr{E}(A), X), & \tau=\infty
\end{array}\right.
$$

with $E(t, x ; \mathscr{E}(A), X)=\inf \left\{\left\|x-x^{0}\right\|: x^{0} \in \mathscr{E}(A),\left|x^{0}\right|_{\mathscr{E}(A)} \leq t\right\}$ for all $x \in X$ and $t>0$.

We will use the real interpolation method. Recall that for a pair of quasi-normed spaces $\left(X_{0},|\cdot| X_{0}\right),\left(X_{1},|\cdot|_{X_{1}}\right)$ and $0<\theta<1,1 \leq q<\infty$ the interpolation space 
generated by $K$-functional is defined as

$$
\left(X_{0}, X_{1}\right)_{\theta, q}=\left\{x \in X_{0}+X_{1}:|x|_{\left(X_{0}, X_{1}\right)_{\theta, q}}<\infty\right\}
$$

with $|x|_{\left(X_{0}, X_{1}\right)_{\theta, q}}=\left(\int_{0}^{\infty}\left[t^{-\theta} K\left(t, x ; X_{0}, X_{1}\right)\right]^{q} d t / t\right)^{1 / q}$, where $K$-functional is determined to be $K\left(t, x ; X_{0}, X_{1}\right)=\inf _{x=x^{0}+x^{1}}\left(\left|x^{0}\right|_{X_{0}}+t\left|x^{1}\right|_{X_{1}}\right)$ for all $x^{0} \in X_{0}$, $x^{1} \in X_{1}$ and $t>0$ (see, e.g. [2]).

Theorem 1 (a) The quasi-normed space $\mathscr{E}(A)$ is complete.

(b) For $0<\theta<1,1 \leq q<\infty, \tau=\theta q, s=1 / \theta-1$ the following isomorphism holds,

$$
\mathscr{B}_{\tau}^{s}(A)=(\mathscr{E}(A), X)_{\theta, q}^{1 / \theta},
$$

where $(\mathscr{E}(A), X)_{\theta, q}$ is the interpolation space determined by $K$-method, and $(\mathscr{E}(A), X)_{\theta, q}^{1 / \theta}$ is defined to be $(\mathscr{E}(A), X)_{\theta, q}$ endowed with the quasi-norm $|x|_{(\mathscr{E}(A), X)_{\theta, q}}^{1 / \theta}$.

Proof (a) By definition $\|x\|_{v}=v \hat{x}(v)$ for each $x \in \mathscr{E}^{v}(A)$ with a given $v>0$, where $\hat{x}(z)=L[x(t)]=\int_{0}^{\infty} x(t) \exp (-z t) d t$ with $\left\{z \in \mathbb{C}:|\operatorname{Re} z|>h^{x}\right\}$ is Laplace's transform of the entire function $x(\lambda)$ in variable $\lambda \in \mathbb{C}$ of an exponential type $h^{x}<v$.

Let $\left(x_{n}\right)$ be a fundamental sequence in $\mathscr{E}(A)$. It is uniformly bounded, i.e., $\exists v>0$ : $\left|x_{n}\right|_{\mathscr{E}(A)}<v$ for all $n \in \mathbb{N}$. Since inf $\left\{\mu:\left(x_{n}\right) \subset \mathscr{E}^{\mu}(A)\right\}<v$, we have $\left(x_{n}\right) \subset \mathscr{E}^{v}(A)$. Thus, the sequence $\left\{[0, \infty) \ni t \mapsto x_{n}(t) \exp (-t v): n \in \mathbb{N}\right\}$ is uniformly absolutely bounded by a constant $K_{v}>0$ for all $t \geq 0$.

By Bernstein's compactness theorem [15, Thm 3.3.6] there exists a convergent subsequence $\left\{x_{n_{i}}(t) \exp (-t v): i \in \mathbb{N}\right\}$ with respect to the topology of uniform convergence in variable $t \in[0, r]$ for all $r>0$. Thus, $\forall \varepsilon>0, \exists n_{\varepsilon} \in \mathbb{N}$ : $\sup _{t \in\left[0, r_{\varepsilon}\right]}\left|x_{n_{i}}(t)-x_{m_{i}}(t)\right| \exp (-t v)<\varepsilon, \forall n_{i}, m_{i} \geq n_{\varepsilon}$, where $r=r_{\varepsilon}>0$ is chosen so large that $K_{\nu} \exp \left(-r_{\varepsilon} v\right)<\varepsilon$. Therefore,

$$
\begin{aligned}
\left\|x_{n_{i}}-x_{m_{i}}\right\|_{2 v} & =2 v L\left[\left|x_{n_{i}}(t)-x_{m_{i}}(t)\right|\right] \\
& =2 v \int_{0}^{\infty}\left|x_{n_{i}}(t)-x_{m_{i}}(t)\right| \exp (-2 t v) d t \\
& =2 v\left(\int_{0}^{r_{\varepsilon}}+\int_{r_{\varepsilon}}^{\infty}\right)\left[\left|x_{n_{i}}(t)-x_{m_{i}}(t)\right| \exp (-t v)\right] \exp (-t v) d t \\
& \leq 2 v \varepsilon \int_{0}^{r_{\varepsilon}} \exp (-t v) d t+2 v K_{v} \int_{r_{\varepsilon}}^{\infty} \exp (-t v) d t \\
& =2 \varepsilon\left[1-\exp \left(-r_{\varepsilon} v\right)\right]+2 K_{v} \exp \left(-r_{\varepsilon} v\right)<4 \varepsilon, \quad n_{i}, m_{i} \geq n_{\varepsilon} .
\end{aligned}
$$

Thus, $\left(x_{n_{i}}\right)$ is fundamental in $\mathscr{E}^{2 v}(A)$. Below, it will be proven that $\mathscr{E}^{2 v}(A)$ is complete. As a conclusion of this, there exists $x_{0} \in \mathscr{E}^{2 v}(A)$ such that $x_{n_{i}} \rightarrow x_{0}$ at $i \rightarrow \infty$ and, consequently, $x_{n} \rightarrow x_{0}$ in $\mathscr{E}^{2 v}(A)$. Hence, $\mathscr{E}(A)$ is complete. 
Now, we prove that $\mathscr{E}^{v}(A)$ with any $v>0$ is complete. Let $\left(x_{n}\right)$ be a fundamental sequence in $\mathscr{E}^{v}(A)$, i.e., $\forall \varepsilon>0 \exists n_{\varepsilon} \in \mathbb{N}:\left\|x_{n}-x_{m}\right\|_{\nu}<\varepsilon$ for all $n, m \geq n_{\varepsilon}$. From $\|x\|_{v} \geq\left\|(A / v)^{k} x\right\|$ with $x \in \mathscr{E}^{v}(A)$ it follows that the sequences $\left(x_{n}\right)$ and $\left((A / v)^{k} x_{n}\right)$ for any $k \in \mathbb{Z}_{+}$are fundamental in $X$. By completeness of $X$ and closeness of $A^{k}$ there exist $x, y_{k} \in X$ such that $x_{n} \rightarrow x$ and $(A / v)^{k} x_{n} \rightarrow y_{k}$ in $X$. So, $y_{k}=(A / v)^{k} x$ with $x \in \mathfrak{D}^{k}(A)$ for all $k \in \mathbb{Z}_{+}$. Thus, $x \in \mathfrak{D}^{\infty}(A)$ and $(A / v)^{k} x_{n} \rightarrow(A / v)^{k} x$ in $X$ as $n \rightarrow \infty$ for all $k \in \mathbb{Z}_{+}$.

Taking in $X$ the limit of $\left\|x_{n}\right\|_{v} \leq\left\|x_{n}-x_{n_{\varepsilon}}\right\|_{\nu}+\left\|x_{n_{\varepsilon}}\right\|_{v} \leq \varepsilon+\left\|x_{n_{\varepsilon}}\right\|_{v}$ as $n \rightarrow \infty$, we find $\|x\|_{\nu} \leq\left\|x_{n_{\varepsilon}}\right\|_{\nu}+\varepsilon$, that is, $x \in \mathscr{E}^{v}(A)$, since $x_{n_{\varepsilon}} \in \mathscr{E}^{v}(A)$. Similarly, from $\left\|x_{n}-x_{m}\right\|_{\nu}<\varepsilon$ as $m \rightarrow \infty$, we find that $\left\|x_{n}-x\right\|_{\nu} \leq \varepsilon$ for all $n \geq n_{\varepsilon}$. Hence, $\mathscr{E} v(A)$ is complete.

b) Now, taking into account the completeness of $\mathscr{E}(A)$ and the continuous embed$\operatorname{ding} \mathscr{E}(A) \leftrightarrow X$, the required equality $\mathscr{B}_{\tau}^{S}(A)=(\mathscr{E}(A), X)_{\theta, q}^{1 / \theta}$ directly follows from [2, Thm 7.1.7]. The proof is completed.

Remark 1 Note that if $A$ is the closure of the differentiation operator $D$ in $X=L_{p}(\mathbb{R})$ with $1<p \leq \infty$ then $\mathscr{B}_{\tau}^{s}(A)$ exactly coincides with the classic Besov space $B_{p, \tau}^{s}(\mathbb{R})$ [6, Thm 7].

\section{Analytical Estimates of Best Approximation Errors}

The analytical estimates of approximation errors are based on exact values of constants. In direct and inverse approximation theorems this problem is solved by exact values of constants in the Bernstein-Jackson inequalities, presented in the following statements.

Theorem 2 Let $0<s<\infty, 0<\tau \leq \infty$ and $\kappa_{s, \tau}:=\left(\tau s^{-1}(s+1)^{2}\right)^{1 / \tau}$.

(a) For each $x \in \mathscr{E}(A)$ the Bernstein-type inequality holds,

$$
|x|_{\mathscr{B}_{\tau}^{s}(A)} \leq c_{s, \tau}|x|_{\mathscr{E}(A)}^{s}\|x\| \text { where } c_{s, \tau}:= \begin{cases}\kappa_{s, \tau}: \tau<\infty \\ 1: & \tau=\infty\end{cases}
$$

(b) For each $x \in \mathscr{B}_{\tau}^{s}(A)$ the following Jackson-type inequality holds,

$$
t^{s} E(t, x ; \mathscr{E}(A), X) \leq 2^{s+1} C_{s, \tau}|x|_{\mathscr{B}_{\tau}^{s}(A)}, \quad C_{s, \tau}:= \begin{cases}\kappa_{s, \tau}^{-1}: & \tau<\infty \\ 2^{-s-1}: \tau=\infty\end{cases}
$$

Herewith, for a fixed s, we have

$$
\liminf _{\tau \rightarrow \infty} \kappa_{s, \tau}=\limsup _{\tau \rightarrow \infty} \kappa_{s, \tau}^{-1}=1
$$

Proof (a) Let $1 \leq q<\infty$ and $x \neq 0$. Since $K(t, x ; \mathscr{E}(A), X) \leq \min \left(|x|_{\mathscr{E}(A)}, t\|x\|\right)$, we obtain

$$
|x|_{(\mathscr{E}(A), X)_{\theta, q}}^{q} \leq\|x\|^{q} \int_{0}^{\alpha} t^{-1+q(1-\theta)} d t+|x|_{\mathscr{E}(A)}^{q} \int_{\alpha}^{\infty} t^{-1-\theta q} d t
$$




$$
\begin{aligned}
& =\frac{1}{q(1-\theta)} \alpha^{q(1-\theta)}\|x\|^{q}+\frac{1}{\theta q} \alpha^{-\theta q}|x|_{\mathscr{E}(A)}^{q} \\
& =\frac{1}{q \theta(1-\theta)}\left(|x|_{\mathscr{E}(A)}^{1-\theta}\|x\|^{\theta}\right)^{q}
\end{aligned}
$$

with $\alpha=|x|_{\mathscr{E}(A)} /\|x\|$. It can be rewritten as

$$
|x|_{(\mathscr{E}(A), X)_{\theta, q}} \leq[q \theta(1-\theta)]^{-1 / q}|x|_{\mathscr{E}(A)}^{1-\theta}\|x\|^{\theta}
$$

If $q=\infty$, we have $K(t, x ; \mathscr{E}(A), X) \leq t^{\theta}|x|_{\mathscr{E}(A)}^{1-\theta}\|x\|^{\theta}$. By combining the previous inequalities,

$$
|x|_{(\mathscr{E}(A), X)_{\theta, q}} \leq \begin{cases}{[q \theta(1-\theta)]^{-1 / q}|x|_{\mathscr{E}(A)}^{1-\theta}\|x\|^{\theta}:} & q<\infty \\ |x|_{\mathscr{E}(A)}^{1-\theta}\|x\|^{\theta}: & q=\infty .\end{cases}
$$

Consider the functional $K_{\infty}(t, x ; \mathscr{E}(A), X):=\inf _{x=x^{0}+x^{1}} \max \left(\left|x^{0}\right| \mathscr{E}(A), t\left\|x^{1}\right\|\right)$. Note that $v^{-\theta} K_{\infty}(v, x ; \mathscr{E}(A), X) \rightarrow 0$ at $v \rightarrow 0$ or $v \rightarrow \infty$, as well as, $t^{s} E(t, x ; \mathscr{E}(A), X) \rightarrow 0$ at $t \rightarrow 0$ or $t \rightarrow \infty$. Integrating by parts with the change of variables $v=t / E(t, x ; \mathscr{E}(A), X)$, we get

$$
\begin{aligned}
\int_{0}^{\infty} & \left(v^{-\theta} K_{\infty}(v, x ; \mathscr{E}(A), X)\right)^{q} d v / v \\
& =-\frac{1}{\theta q} \int_{0}^{\infty} K_{\infty}(v, x ; \mathscr{E}(A), X)^{q} d v^{-\theta q} \\
& =\frac{1}{\theta q} \int_{0}^{\infty} v^{-\theta q} d K_{\infty}(v, x ; \mathscr{E}(A), X)^{q}=\frac{1}{\theta q} \int_{0}^{\infty}(t / E(t, x ; \mathscr{E}(A), X))^{-\theta q} d t^{q} \\
& =\frac{1}{\theta q^{2}} \int_{0}^{\infty}\left(t^{s} E(t, x ; \mathscr{E}(A), X)\right)^{\theta q} d t / t \quad \text { with } \quad s=1 / \theta-1
\end{aligned}
$$

The following inequalities are a consequence of definitions $K$ and $K_{\infty}[16$, Rem. $3.1]$,

$$
K_{\infty}(t, x ; \mathscr{E}(A), X) \leq K(t, x ; \mathscr{E}(A), X) \leq 2 K_{\infty}(t, x ; \mathscr{E}(A), X)
$$

According to the left inequality from (5), we have 


$$
\begin{aligned}
\frac{1}{\theta q^{2}}|x|_{\mathscr{B}_{\tau}^{s}(A)}^{\theta q} & =\frac{1}{\theta q^{2}} \int_{0}^{\infty}\left(t^{s} E(t, x ; \mathscr{E}(A), X)\right)^{\theta q} d t / t \\
& =\int_{0}^{\infty}\left(v^{-\theta} K_{\infty}(v, x ; \mathscr{E}(A), X)\right)^{q} d v / v \\
& \leq \int_{0}^{\infty}\left(v^{-\theta} K(v, x ; \mathscr{E}(A), X)\right)^{q} d v / v=|x|_{(\mathscr{E}(A), X)_{\theta, q}}^{q}
\end{aligned}
$$

On the other hand, from the right inequality (5) it follows that

$$
\begin{aligned}
|x|_{(\mathscr{E}(A), X)_{\theta, q}}^{q} & =\int_{0}^{\infty}\left(v^{-\theta} K(v, x ; \mathscr{E}(A), X)\right)^{q} d v / v \\
& =2^{q} \int_{0}^{\infty}\left(v^{-\theta} K_{\infty}(v, x ; \mathscr{E}(A), X)\right)^{q} d v / v \\
& =2^{q} \frac{1}{\theta q^{2}} \int_{0}^{\infty}\left(t^{s} E(t, x ; \mathscr{E}(A), X)\right)^{\theta q} d t / t=2^{q} \frac{1}{\theta q^{2}}|x|_{\mathscr{B}_{\tau}^{s}(A)}^{\theta q}
\end{aligned}
$$

Thus, combining the previous inequalities, we get

$$
|x|_{(\mathscr{E}(A), X)_{\theta, q}}^{q} \leq 2^{q}\left(\theta q^{2}\right)^{-1}|x|_{\mathscr{B}_{\tau}^{s}(A)}^{\theta q} \leq 2^{q}|x|_{(\mathscr{E}(A), X)_{\theta, q}}^{q} \text { with } \tau=\theta q
$$

Via [2, Lemma 7.1.2] for every $v>0$ there exists $t>0$ such that

$$
\begin{aligned}
\left.t^{s} E(t, x ; \mathscr{E}(A), X)\right)^{\theta} & \leq v^{-\theta} K_{\infty}(v, x ; \mathscr{E}(A), X) \\
& \leq\left(t^{s} E(t-0, x ; \mathscr{E}(A), X)\right)^{\theta}
\end{aligned}
$$

Since $|x|_{\mathscr{B}_{\infty}^{s}(A)}^{\theta} \leq|x|_{(\mathscr{E}(A), X)_{\theta, \infty}}$, the inequalities (7) yield

$$
\begin{aligned}
v^{-\theta} K(v, x ; \mathscr{E}(A), X) & \leq v^{-\theta} 2 K_{\infty}(v, x ; \mathscr{E}(A), X) \leq 2\left(t^{s} E(t-0, x ; \mathscr{E}(A), X)\right)^{\theta} \\
& \leq 2\left(\sup _{t>0} t^{s} E(t, x ; \mathscr{E}(A), X)\right)^{\theta}=2|x|_{\mathscr{B}_{\infty}^{s}(A)}^{\theta}
\end{aligned}
$$

As result, $|x|_{(\mathscr{E}(A), X)_{\theta, \infty}} \leq 2|x|_{\mathscr{B}_{\infty}^{s}(A)}^{\theta}$. By applying (4), we obtain

$$
|x|_{\mathscr{B}_{\tau}^{s}(A)}^{\theta} \leq \begin{cases}{[q /(1-\theta)]^{1 / q}|x|_{\mathscr{E}(A)}^{1-\theta}\|x\|^{\theta}:} & q<\infty \\ |x|_{\mathscr{E}(A)}^{1-\theta}\|x\|^{\theta}: & q=\infty .\end{cases}
$$

Setting $s=1 / \theta-1$ and $\tau=\theta q$ in (8), we get the inequalities (1).

b) By integration both sides of $\min (1, v / t) K(t, x ; \mathscr{E}(A), X) \leq K(v, x ; \mathscr{E}(A), X)$, we get 


$$
\begin{aligned}
& \left(\int_{0}^{\infty}\left(v^{-\theta} \min (1, v / t)\right)^{q} \frac{d v}{v}\right)^{1 / q} K(t, x ; \mathscr{E}(A), X) \\
& \quad \leq\left(\int_{0}^{\infty}\left(v^{-\theta} K(v, x ; \mathscr{E}(A), X)\right)^{q} \frac{d v}{v}\right)^{1 / q}=|x|_{(\mathscr{E}(A), X)_{\theta, q}} \text { and } \\
& \left(\int_{0}^{\infty}\left(v^{-\theta} \min (1, v / t)\right)^{q} \frac{d v}{v}\right)^{1 / q}=\left(\int_{0}^{t} v^{(1-\theta) q-1} t^{-q} d v+\int_{t}^{\infty} v^{-\theta q-1} d v\right)^{1 / q} \\
& =\frac{1}{[q \theta(1-\theta)]^{1 / q} t^{\theta}}
\end{aligned}
$$

respectively. As a result,

$$
\begin{aligned}
& \left(\int_{0}^{t} \frac{v^{(1-\theta) q-1}}{t^{q}} d v+\int_{t}^{\infty} v^{-\theta q-1} d v\right)^{1 / q} K(t, x ; \mathscr{E}(A), X) \\
& \quad=\frac{K(t, x ; \mathscr{E}(A), X)}{[q \theta(1-\theta)]^{1 / q} t^{\theta}} \leq|x|_{(\mathscr{E}(A), X)_{\theta, q}}
\end{aligned}
$$

Hence, $K(t, x ; \mathscr{E}(A), X) \leq[q \theta(1-\theta)]^{1 / q} t^{\theta}|x|_{(\mathscr{E}(A), X)_{\theta, q}}$. Taking into account (5), (7), we get

$v^{1-\theta} E(v, x ; \mathscr{E}(A), X)^{\theta} \leq t^{-\theta} K_{\infty}(t, x ; \mathscr{E}(A), X) \leq[q \theta(1-\theta)]^{1 / q}|x|_{(\mathscr{E}(A), X)_{\theta, q}}$.

Applying (6), we obtain $v^{1-\theta} E(v, x ; \mathscr{E}(A), X)^{\theta} \leq 2[(1-\theta) / q]^{1 / q}|x|_{\mathscr{B}_{\tau}^{s}(A)}^{\theta}$. Setting $s=(1-\theta) / \theta$ and $\tau=\theta q$, we get (2) in the case $1 \leq q<\infty$. In the case $q=\infty$, we have

$$
t^{s} E(t, x ; \mathscr{E}(A), X) \leq \sup _{t>0} t^{s} E(t, x ; \mathscr{E}(A), X)=|x|_{\mathscr{B}_{\infty}^{s}(A)}
$$

for all $x \in \mathscr{B}_{\infty}^{S}(A)$. Thus, the inequalities (2) hold for both cases that ends the proof. The limits (3) are calculated directly.

Remark 2 The known values of constants in the Bernstein-Jackson inequalities for some particular cases can be found in [11, pp. 257-259], [10, Thm 1], [19, p. 595] and in others.

\section{Conditions for Non-triviality of Approximation Scales}

We give a simple criterion that, in the case of self-adjoint operators, ensures the equality of $\mathscr{E}(A)$ with all spectral subspaces of $A$.

Proposition 1 If a strongly continuous group $\mathbb{R} \ni t \rightarrow e^{\dot{\mathrm{i}} t A}$ on $X$, generated by $\dot{\mathrm{i}} A$, is such that $\lim \sup _{|t| \rightarrow \infty}\left\|e^{\dot{\mathrm{i}} t A} x\right\|=M_{x}<\infty$ for all $x \in X$ then the embedding $\mathscr{E}(A) \leftrightarrow X$ is dense. 
Proof Let a function $f \in L_{1}(\mathbb{R})$ be the restriction to $\mathbb{R}$ of an entire function of exponential type $\tau>0$ such that $\int_{-\infty}^{\infty} f(t) d t=1$ and $P_{\alpha}=\alpha \int_{-\infty}^{\infty} f(\alpha t) e^{\dot{\mathrm{i}} t A} d t$ with $\alpha>0$. There exists $c>0$ independent on $k$ such that Bernstein's inequality $\int_{-\infty}^{\infty}\left|f^{(k)}(t)\right| d t \leq c \tau^{k}$ holds for all $k \in \mathbb{Z}_{+}[15$, p. 115]. From the known relation $\lim _{|t| \rightarrow \infty}\left|f^{(k)}(t)\right|=0$ (see [15, Thm 3.2.5]) via integration by parts, we have

$$
(\dot{\mathrm{i}} A)^{k} P_{k} x=\alpha \int_{-\infty}^{\infty} f(\alpha t)(\dot{\mathrm{i}} A)^{k} e^{\dot{\mathrm{i}} t A} x d t=\alpha^{k} \int_{-\infty}^{\infty} f^{(k)}(t) e^{\dot{\mathrm{i}} t / \alpha A} x d t, \quad x \in X .
$$

By Bernstein's inequality it follows, that $\left\|A^{k} P_{\alpha} x\right\| \leq c\|x\|(\tau \alpha)^{k}$ for all $x \in X$. Consequently, $\left\|A^{k} P_{\alpha} x\right\|^{1 / k} \leq(c\|x\|)^{1 / k} \tau \alpha$ and $\lim ^{1 / k} \sup _{k \rightarrow \infty}\left\|A^{k} P_{\alpha} x\right\|^{1 / k} \leq \tau \alpha$. If $\nu>\tau \alpha$ then $P_{\alpha} x \in \mathscr{E}^{\nu}(A)$ for all $x \in X$. Hence, $\bigcup_{\alpha>0}\left\{P_{\alpha} x: x \in X\right\} \subset \mathscr{E}(A)$.

Using the equality $P_{\alpha} x-x=\alpha \int_{-\infty}^{\infty} f(\alpha t)\left(e^{\dot{1} t} A x-x\right) d t$, we show that

$$
\lim _{\alpha \rightarrow \infty}\left\|P_{\alpha} x-x\right\|=0, \quad x \in X
$$

Since the $X$-valued function $\mathbb{R} \ni t \rightarrow e^{\dot{\mathrm{i}} t} A x-x$ is continuous at $t=0$, for every $\varepsilon>0$ there exists $\delta>0$ such that $\max _{|t| \leq \delta}\left\|e^{\dot{\mathrm{u}} t A} x-x\right\| \leq \varepsilon$. Therefore,

$$
\begin{aligned}
\left\|P_{\alpha} x-x\right\| & \leq \varepsilon \int_{|t| \leq \delta}|\alpha f(\alpha t)| d t+\int_{|t|>\delta}|\alpha f(\alpha t)|\left\|e^{\dot{\mathrm{i}} t A} x-x\right\| d t \\
& \leq \varepsilon \int_{|t| \leq \delta \alpha}|f(t)| d t+\max _{|t|>\delta \alpha}\left\|e^{\dot{\mathrm{i}} t A} x-x\right\| \int_{|t|>\delta \alpha}|f(t)| d t .
\end{aligned}
$$

Since lim $\sup _{|t| \rightarrow \infty}\left\|e^{\mathrm{i} t A} x-x\right\| \leq M_{x}+\|x\|<\infty$ for all $x \in X$, we obtain

$$
\lim _{\alpha \rightarrow \infty}\left\|P_{\alpha} x-x\right\| \leq \varepsilon\|f\|_{L_{1}(\mathbb{R})}+\left(M_{x}+\|x\|\right) \lim _{\alpha \rightarrow \infty} \int_{|t|>\delta \alpha}|f(t)| d t=\varepsilon\|f\|_{L_{1}(\mathbb{R})} .
$$

Since $\varepsilon$ is arbitrary, (9) holds. Hence, $\bigcup_{\alpha>0}\left\{P_{\alpha} x: x \in X\right\}$ and therefore $\mathscr{E}(A)$ are dense in $X$ what ends the proof.

Remark 3 If $A$ is self-adjoint in a Hilbert space $X$ then the group $e^{\dot{\mathrm{i}} t A}$ is unitary by Stone's theorem. Thus, in this case $M_{x} \equiv 1$ and the embedding $\mathscr{E}(A) \rightarrow X$ is dense. Approximation problems for cases of self-adjoint operators $A$ was analyzed in [10] where, instead of the Besov-type quasi-norm, the smoothness modulus is used.

\section{Applications to Elliptic Operators on Bounded Domains with Smooth Coefficients}

Note that a simple case of elliptic operators with constant coefficients was considered in [6]. Now, we adapt the Bernstein-Jackson inequalities (1-2) to the case of regular elliptic operators with variable smooth coefficients. 
We will need one general result obtained earlier in [5]. Suppose that $A$ has a discrete spectrum $\sigma(A)$, i.e., its resolvent $R(\lambda, A)=(\lambda-A)^{-1}$ has only isolated eigenvalues $\left\{\lambda_{j} \in \mathbb{C}: j \in \mathbb{N}\right\}$ of finite multiplicities which are poles with the limit at infinity. In particular, this guarantees the compactness of $R(\lambda, A)$ (see, e.g. [12, p.187]). Let $\mathscr{R}_{\lambda_{j}}(A)=\left\{x \in \mathfrak{D}^{\infty}(A):\left(\lambda_{j}-A\right)^{r_{j}} x=0\right\}$ be the spectral subspace, corresponding to the eigenvalue $\lambda_{j}$ of multiplicity $r_{j}$. Denote by $\mathscr{R}^{v}(A)$ the complex linear span in $X$ of all spectral subspaces $\mathscr{R}_{\lambda_{j}}(A)$ such that $\left|\lambda_{j}\right|<v$. Let us define on $\mathscr{R}(A):=$ $\bigcup_{v>0} \mathscr{R}^{v}(A)$ the quasi-norm

$$
|x|_{\mathscr{R}(A)}=\|x\|+\inf \left\{v>0: x \in \mathscr{R}^{v}(A)\right\} .
$$

In [5, Thm 2.2] it is proved that the following equalities hold,

$$
\mathscr{E}(A)=\mathscr{R}(A), \quad|x|_{\mathscr{E}(A)}=|x|_{\mathscr{R}(A)} \quad(\forall x \in \mathscr{E}(A))
$$

As a consequence, in the case of operators $A$ with discrete spectrum, Theorem 2 can be slightly strengthened. Namely (see [6, Thm 6]), the following inequalities hold,

$$
\begin{aligned}
|x|_{\mathscr{B}_{\tau}^{s}(A)} & \leq c_{s, \tau}|x|_{\mathscr{R}(A)}^{s}\|x\|, \quad u \in \mathscr{R}(A), \\
\inf \left\{\left\|x-x^{0}\right\|: x^{0} \in \mathscr{R}^{\nu}(A)\right\} & \leq v^{-s} 2^{s+1} C_{s, \tau}|x|_{\mathscr{B}_{\tau}^{s}(A)}, \quad x \in \mathscr{B}_{\tau}^{s}(A)
\end{aligned}
$$

with the constants $c_{s, \tau}$ and $C_{s, \tau}$ from Theorem 2 .

Consider the space $L_{p}(\Omega),(1<p \leq \infty)$ on $\Omega \subset \mathbb{R}^{n}$. The Sobolev space $W_{p}^{m}(\Omega)$ has norm $\|u\|_{W_{p}^{m}(\Omega)}=\sum_{|\alpha| \leq m}\left\|D^{\alpha} u\right\|_{L_{p}(\Omega)}, \alpha=\left(\alpha_{1}, \ldots, \alpha_{n}\right) \in$ $\mathbb{N}^{n},|\alpha|=\alpha_{1}+\cdots+\alpha_{n}$, where $D$ is differentiation. The asymptotic equality $K\left(t^{m}, u, W_{p}^{m}(\Omega), L_{p}(\Omega)\right) \asymp \omega_{m}(u, t)_{L_{p}}$ holds, where $\omega_{m}(u, t)_{L_{p}}:=\sup _{|h| \leq t} \| \Delta_{h}^{m}$ $(u, \cdot) \|_{L_{p}(\Omega)}$ is the $m$ th order modulus of smoothness for $u \in L_{p}(\Omega)$ and $\Delta_{h}^{m}(u, t):=$ $\sum_{k=0}^{m}(-1)^{m-k}\left(\begin{array}{c}m \\ k\end{array}\right) u(t+k h)$ is the $m$ th difference with step $h$. Take $m=[s]+1$ (the smallest integer larger than $s)$, then the classic Besov space $B_{p, \tau}^{s}(\Omega)$ can be endowed with the norm (see e.g., $[4,16]$ )

$$
\|u\|_{B_{p, \tau}^{s}(\Omega)}=\left\{\begin{array}{lr}
\left(\int_{0}^{\infty}\left[t^{-s} \omega_{[s]+1}(u, t)_{L_{p}}\right]^{\tau} \frac{d t}{t}\right)^{1 / \tau}, & 0<\tau<\infty \\
\sup _{t>0} t^{-s} \omega_{[s]+1}(u, t)_{L_{p}}, & \tau=\infty
\end{array}\right.
$$

Now, let $A$ be a closed linear operator in the space $L_{p}(\Omega)$ over an open bounded set $\Omega \subset \mathbb{R}^{n}$ with infinitely smooth boundary $\partial \Omega$, which is determined on the domain $W_{p, A}^{2 m}(\Omega)=\left\{u \in W_{p}^{2 m}(\Omega):\left.b_{j} u\right|_{\partial \Omega}=0, j=1, \ldots, m\right\}$ via the regular elliptic system [18, Def. 5.2.1/4] 


$$
\begin{aligned}
& (A u)(\xi)=\sum_{|\alpha| \leq 2 m} a_{\alpha}(\xi) D^{\alpha} u(\xi), \quad a_{\alpha} \in C^{\infty}(\bar{\Omega}), \quad \bar{\Omega}=\Omega \cup \partial \Omega, \\
& \left(b_{j} u\right)(\xi)=\sum_{|\alpha| \leq m_{j}} b_{j, \alpha}(\xi) D^{\alpha} u(\xi), \quad b_{j, \alpha} \in C^{\infty}(\partial \Omega), j=1, \ldots, m .
\end{aligned}
$$

We assume that its resolvent set $\rho(A)$ is non empty. This is enough for the compactness of $R(\lambda, A)$ for any $\lambda \in \rho(A)$ and the closeness for all integer powers $A^{k}$. Thus, the spectrum $\sigma(A)$ is discrete and is independent on $p$ [18, Sec. 5.4.4]. Let $0 \in \rho(A)$ for simplicity.

Let $0<s<\infty, 1<p<\infty, 1 \leq \tau \leq \infty$. In the Besov space $B_{p, \tau}^{s}(\Omega)$ we consider the subspace which is associated with the operator $A$ (see [18, Def. 4.2.1/1]),

$$
B_{p, \tau, A}^{s}(\Omega):=\left\{u \in B_{p, \tau}^{s}(\Omega):\left.b_{j} A^{k} u\right|_{\partial \Omega}=0, j=1, \ldots, m, k \in \mathbb{Z}_{+}\right\} .
$$

Theorem 3 The following Bernstein-Jackson inequalities hold,

$$
\begin{aligned}
\|u\|_{B_{p, \tau}^{s}(\Omega)} & \leq c_{s, \tau}|u|_{\mathscr{R}(A)}^{s}\|u\|_{L_{p}(\Omega)}, \quad u \in \mathscr{R}(A), \\
t^{s} E\left(t, u ; \mathscr{R}(A), L_{p}(\Omega)\right) & \leq 2^{s+1} C_{s, \tau}\|u\|_{B_{p, \tau}^{s}(\Omega)}, \quad u \in B_{p, \tau, A}^{s}(\Omega)
\end{aligned}
$$

with the constants $c_{s, \tau}$ and $C_{s, \tau}$ from Theorem 2, where is denoted $E(t, u ; \mathscr{R}(A)$, $\left.L_{p}(\Omega)\right)=\inf \left\{\left\|u-u^{0}\right\|_{L_{p}(\Omega)}: u^{0} \in \mathscr{R}(A),\left|u^{0}\right| \mathscr{R}(A) \leq t\right\}$ for all $u \in L_{p}(\Omega)$. In addition, for each functions $u \in B_{p, \tau, A}^{s}(\Omega)$ the following inequality holds,

$$
\inf \left\{\left\|u-u^{0}\right\|_{L_{p}(\Omega)}: u^{0} \in \mathscr{R}^{v}(A)\right\} \leq v^{-s} 2^{s+1} C_{s, \tau}\|u\|_{B_{p, \tau}^{s}(\Omega)} .
$$

Proof Let the space $\mathscr{E}^{v}(D)=\left\{u \in C^{\infty}(\bar{\Omega}): D^{\alpha} u \in L_{p}(\Omega),|\alpha|=k \in \mathbb{Z}_{+}\right\}$be endowed with the norm $\|u\|_{\mathscr{E}^{\nu}(D)}=\sum_{k \geq 0} \sum_{|\alpha|=k} v^{-k}\left\|D^{\alpha} u\right\|_{L_{p}(\Omega)}$. On $\mathscr{E}(D)=$ $\bigcup_{v>0} \mathscr{E}^{v}(D)$ we define the quasinorm $|u|_{\mathscr{E}(D)}=\|u\|_{L_{p}(\Omega)}+\inf \left\{v>0: u \in \mathscr{E}^{v}(D)\right\}$.

First, we show that for any $a_{\alpha} \in C^{\infty}(\bar{\Omega})$ the following equality holds,

$$
\mathscr{B}_{\tau}^{s}\left(A, L_{p}(\Omega)\right)=B_{p, \tau, A}^{s}(\Omega)
$$

where $\mathscr{B}_{\tau}^{s}\left(A, L_{p}(\Omega)\right):=\mathscr{B}_{\tau}^{s}(A)$ with $X=L_{p}(\Omega)$. It is enough to prove the equality

$$
\mathscr{E}(A)=\left\{u \in \mathscr{E}(D):\left.b_{j} A^{k} u\right|_{\partial \Omega}=0, j=1, \ldots, m, k \in \mathbb{Z}_{+}\right\}
$$

Since $\left\|A^{k} u\right\|_{L_{p}(\Omega)} \leq v^{k}\|u\|_{L_{p}(\Omega)} \leq v^{2 k}\left(\sum_{|\alpha|=k} v^{-k}\left\|D^{\alpha} u\right\|_{L_{p}(\Omega)}+v^{-k}\|u\|_{L_{p}(\Omega)}\right)$ for all $u \in \mathscr{E}^{\mathscr{v}}(A)$, we get $\sum v^{-2 k}\left\|A^{k} u\right\|_{L_{p}(\Omega)} \leq \sum\left(\sum_{|\alpha|=k} v^{-k}\left\|D^{\alpha} u\right\|_{L_{p}(\Omega)}+\right.$ $\left.v^{-k}\|u\|_{L_{q}(\Omega)}\right)$. Substituting $\mu=v^{2}$ with $v>1$, we have

$$
\|u\|_{\mu} \leq\|u\|_{\mathscr{E}^{\nu}(D)}+\frac{v\|u\|_{L_{p}(\Omega)}}{v-1} \leq\|u\|_{\mathscr{E}^{\nu}(D)}+\frac{v\|u\|_{\mathscr{E}^{\nu}(D)}}{v-1}=\frac{2 v-1}{v-1}\|u\|_{\mathscr{E}^{\nu}(D)}
$$


It directly follows that $\left\{u \in \mathscr{E}^{\sqrt{v}}(D):\left.b_{j} A^{k} u\right|_{\partial \Omega}=0, j=1, \ldots, m, k \in \mathbb{Z}_{+}\right\} \subset$ $\mathscr{E}^{\mathrm{V}}(A)$.

On the other hand, according to [18, Thm 5.4.3] for any $k \in \mathbb{N}$ there exists $c_{k}>0$ such that $\left\|A^{k} u\right\|_{L_{p}(\Omega)} \geq c_{k}\|u\|_{W_{p}^{2 m k}(\Omega)}$ for all $u \in \mathfrak{D}^{k}(A)$. Thus,

$$
\begin{aligned}
\left\|A^{k+1} u\right\|_{L_{p}(\Omega)} & =\left\|A^{k}(A u)\right\|_{L_{p}(\Omega)} \geq c_{k}\|A u\|_{W_{p}^{2 m k}(\Omega)}=c_{k} \sum_{|\alpha| \leq 2 m k}\left\|D^{\alpha} A u\right\|_{L_{p}(\Omega)} \\
& \geq c_{k} \sum_{|\alpha| \leq 2 m k}\left\|A D^{\alpha} u\right\|_{L_{p}(\Omega)} \geq c_{k} c_{1} \sum_{|\alpha| \leq 2 m k}\left\|D^{\alpha} u\right\|_{W_{p}^{2 m}(\Omega)} \\
& =c_{k+1}\|u\|_{W_{p}^{2 m(k+1)}(\Omega)}
\end{aligned}
$$

where $c_{k+1}=c_{k} c_{1}=c_{1}^{k}$ by induction on $k$. Hence, for each $k \in \mathbb{Z}_{+}$and $u \in \mathfrak{D}^{k}(A)$, we have $\left\|A^{k} u\right\|_{L_{p}(\Omega)} \geq c_{1}^{k}\|u\|_{W_{p}^{2 m k}(\Omega)}$ for all $u \in \mathfrak{D}^{k}(A)$, where $c_{1}>0$ does not depend on $k$. This leads to the inequality $\sum v^{-k}\left\|A^{k} u\right\|_{L_{p}(\Omega)} \geq \sum\left(c_{1}^{-1} v\right)^{-k}\|u\|_{W_{p}^{k}(\Omega)}$ from which it follows that

$$
\mathscr{E}^{v}(A) \subset\left\{u \in \mathscr{E}^{c_{1}^{-1} v}(D):\left.b_{j} A^{k} u\right|_{\partial \Omega}=0, j=1, \ldots, m, k \in \mathbb{Z}_{+}\right\}
$$

Hence, the equality (16) holds.

Applying now Theorem 2 and (10), as well as, taking into account (16), we obtain the required inequalities (12-13), while (14) directly follows from (11).

\section{Cases of Ordinary Differential Operators}

Set $-\infty<a<b<\infty$. Let $B_{2, \tau}^{s}(\Omega)$ be the classical Besov space on $\Omega=(a, b)$ and a function $p \in C^{\infty}(\bar{\Omega})$ is such that $p(\xi)>0(\xi \in \Omega), 0<C_{a}=\lim _{\xi \downarrow a} \frac{p(\xi)}{\xi-a}<\infty$, $0<C_{b}=\lim _{\xi \uparrow b} \frac{p(\xi)}{b-\xi}<\infty$. Let us define the Legendre operators

$$
A_{m, l} u=(-1)^{m} \frac{d^{m}}{d \xi^{m}}\left(p^{l}(\xi) \frac{d^{m} u}{d \xi^{m}}\right), \quad l=0,1, \ldots, m, \quad m=1,2, \ldots
$$

with $\mathfrak{D}\left(A_{m, l}\right)=\left\{u \in C^{\infty}(\bar{\Omega}): u^{(j)}(a)=u^{(j)}(b)=0, j=0, \ldots, m-l-1\right\}$ for all indexes $l=0,1, \ldots, m-1$ where $\mathfrak{D}\left(A_{m, m}\right)=C^{\infty}(\bar{\Omega})$.

From [18, Thm 7.4.1], it follows that $A_{m, l}$ have closures $\bar{A}_{m, l}$ in $L_{2}(\Omega)$ with $\mathfrak{D}\left(\bar{A}_{m, l}\right)=\left\{u \in W_{2}^{2 m}\left(\Omega ; p^{2 l}\right): u^{(j)}(a)=u^{(j)}(b)=0, j=0, \ldots, m-l-1\right\}$ for $l=0,1, \ldots, m-1$. Here $W_{2}^{2 m}\left(\Omega ; p^{2 l}\right)=\left\{u \in L_{2}(\Omega):\|u\|_{W_{2}^{2 m}\left(\Omega ; p^{2 l}\right)}^{2}=\right.$ $\left.\sum_{j=0}^{2 m} \int_{\Omega} p^{2 l}(\xi)\left|u^{(j)}(\xi)\right|^{2} d \xi<\infty\right\}$ and $\mathfrak{D}\left(\bar{A}_{m, m}\right)=W_{2}^{2 m}\left(\Omega ; p^{2 m}\right)$. Then the operators $\bar{A}_{m, l}$ are self-adjoint with discrete $\sigma\left(\bar{A}_{m, l}\right)$ in $L_{2}(\Omega)$ and their sets of exponential type vectors are dense in $L_{2}(\Omega)$ by Proposition 1 . We assume that $0 \in \rho\left(\bar{A}_{m, l}\right)$, oth- 
erwise, we replace $L_{2}(\Omega)$ through the subspace without the finite-dimensional kernel of $\bar{A}_{m, l}$.

Theorem 4 The following Bernstein-Jackson inequalities hold,

$$
\begin{aligned}
\|u\|_{B_{2, \tau}^{s}(\Omega)} & \leq c_{s, \tau}|u|_{\mathscr{R}\left(\bar{A}_{m, l}\right)}^{s}\|u\|_{L_{2}(\Omega)}, \quad u \in \mathscr{R}\left(\bar{A}_{m, l}\right), \\
t^{s} E\left(t, u ; \mathscr{R}\left(\bar{A}_{m, l}\right), L_{2}(\Omega)\right) & \leq 2^{s+1} C_{s, \tau}\|u\|_{B_{2, \tau}^{s}(\Omega)}, \quad u \in \mathscr{B}_{\tau}^{s}\left(\bar{A}_{m, l}, L_{2}(\Omega)\right)
\end{aligned}
$$

with the constants $c_{s, \tau}$ and $C_{s, \tau}$ from Theorem 2, where is denoted

$$
E\left(t, u ; \mathscr{R}\left(\bar{A}_{m, l}\right), L_{2}(\Omega)\right)=\inf \left\{\left\|u-u^{0}\right\|_{L_{2}(\Omega)}: u^{0} \in \mathscr{R}\left(\bar{A}_{m, l}\right),\left|u^{0}\right|_{\mathscr{R}\left(\bar{A}_{m, l}\right)} \leq t\right\}
$$

for all $u \in L_{2}(\Omega)$,

$$
\begin{gathered}
\mathscr{B}_{\tau}^{s}\left(\bar{A}_{m, l}, L_{2}(\Omega)\right)=\left\{u \in B_{2, \tau}^{s}(\Omega):\left(\bar{A}_{m, l}^{k} u\right)^{(j)}(a)=\left(\bar{A}_{m, l}^{k} u\right)^{(j)}(b)=0,\right. \\
\quad j=0, \ldots, m-l-1, k=0,1, \ldots\}, \\
l=0,1, \ldots, m-1, \\
\mathscr{B}_{\tau}^{s}\left(\bar{A}_{m, m}, L_{2}(\Omega)\right)=B_{2, \tau}^{s}(\Omega) .
\end{gathered}
$$

In addition, for all $u \in \mathscr{B}_{\tau}^{s}\left(\bar{A}_{m, l}, L_{2}(\Omega)\right)$ the following inequality holds,

$$
\inf \left\{\left\|u-u^{0}\right\|_{L_{2}(\Omega)}: u^{0} \in \mathscr{R}^{v}\left(\bar{A}_{m, l}\right)\right\} \leq v^{-s} 2^{s+1} C_{s, \tau}|u|_{B_{2, \tau}^{s}(\Omega)}
$$

Proof Let us consider the space $\mathscr{E}^{\nu}(D)=\left\{u \in C^{\infty}(\bar{\Omega}): u^{(k)} \in L_{2}(\Omega), k \in \mathbb{Z}_{+}\right\}$ endowed with the norm $\|u\|_{\mathscr{E}^{v}(D)}=\sum_{k \geq 0} v^{-k}\left\|u^{(k)}\right\|_{L_{2}(\Omega)}$ and set $\mathscr{E}(D)=$ $\bigcup_{v>0} \mathscr{E}^{v}(D)$, where $D$ means the differentiation operator in $L_{2}(\Omega)$. The space $\mathscr{E}(D)$ we endow with the quasi-norm $|u|_{\mathscr{E}(D)}=\|u\|_{L_{2}(\Omega)}+\inf \{v>0: u \in$ $\left.\mathscr{E}^{v}(D)\right\}$. Applying [18, Lemma 7.3.1/1] for all $u \in C^{\infty}(\bar{\Omega})$, we obtain the equivalence $\left\|\bar{A}_{m, l}^{k} u\right\|_{L_{2}(\Omega)}^{2} \asymp\|u\|_{W_{2}^{2 k m}\left(\Omega ; p^{2 k l}\right)}^{2}$. Hence, there exists $c_{k}(l)>0$ such that $\left\|\bar{A}_{m, l}^{k} u\right\|_{L_{2}(\Omega)} \geq c_{k}\left\|u^{(k)}\right\|_{L_{2}(\Omega)}$ for all $u \in \mathfrak{D}^{k}\left(\bar{A}_{m, l}\right)$. Then by iteration

$$
\begin{aligned}
\left\|\bar{A}_{m, l}^{k+1} u\right\|_{L_{2}(\Omega)} & =\left\|\bar{A}_{m, l}^{k}\left(\bar{A}_{m, l} u\right)\right\|_{L_{2}(\Omega)} \geq c_{k}\left\|\left(\bar{A}_{m, l} u\right)^{(k)}\right\|_{L_{2}(\Omega)} \\
& \geq c_{k}\left\|\bar{A}_{m, l} u^{(k)}\right\|_{L_{2}(\Omega)} \geq c_{k+1}\left\|u^{(k+1)}\right\|_{L_{2}(\Omega)}
\end{aligned}
$$

where $c_{k+1}=c_{k} c_{1}=c_{1}^{k}$. Thus, $\left\|\bar{A}_{m, l}^{k} u\right\|_{L_{2}(\Omega)} \geq c_{1}^{k}\left\|u^{(k)}\right\|_{L_{2}(\Omega)}$ for $k \in \mathbb{Z}_{+}$, $u \in \mathfrak{D}^{k}\left(\bar{A}_{m, l}\right)$ with $c_{1}(l)>0$ independent on $k$. This implies that $\sum v^{-k}\left\|\bar{A}_{m, l}^{k} u\right\|_{L_{2}(\Omega)} \geq$ $\sum\left(c_{1}^{-1} v\right)^{-k}\left\|u^{(k)}\right\|_{L_{2}(\Omega)}$. Hence, $\mathscr{E} v\left(\bar{A}_{m, m}\right) \subset \mathscr{E}^{c_{1}^{-1} v}(D)$, as well as,

$$
\begin{gathered}
\mathscr{E}^{v}\left(\bar{A}_{m, l}\right) \subset\left\{u \in \mathscr{E}^{-1} v(D):\left(\bar{A}_{m, l}^{k} u\right)^{(j)}(a)=\left(\bar{A}_{m, l}^{k} u\right)^{(j)}(b)=0,\right. \\
\left.j=0, \ldots, m-l-1, k \in \mathbb{Z}_{+}\right\}, \\
l=0,1, \ldots, m-1 .
\end{gathered}
$$


On the other hand, $\left\|\bar{A}_{m, l}^{k} u\right\|_{L_{2}(\Omega)} \leq v^{k}\|u\|_{L_{2}(\Omega)}$ for all $u \in \mathscr{E}^{v}\left(\bar{A}_{m, l}\right), k \in \mathbb{Z}_{+}$. Thus, $\sum v^{-2 k}\left\|\bar{A}_{m, l}^{k} u\right\|_{L_{2}(\Omega)} \leq \sum v^{-k}\left(\|u\|_{L_{2}(\Omega)}+\left\|u^{(k)}\right\|_{L_{2}(\Omega)}\right)$. It follows that $\mathscr{E}^{\sqrt{v}}(D) \subset \mathscr{E}^{v}\left(\bar{A}_{m, m}\right)$,

$$
\begin{aligned}
\mathscr{E}^{v}\left(\bar{A}_{m, l}\right) \supset\left\{u \in \mathscr{E}^{\sqrt{v}}(D):\left(\bar{A}_{m, l}^{k} u\right)^{(j)}(a)=\left(\bar{A}_{m, l}^{k} u\right)^{(j)}(b)=0,\right. \\
\left.j=0, \ldots, m-l-1, k \in \mathbb{Z}_{+}\right\}, l=0,1, \ldots, m-1 .
\end{aligned}
$$

Applying now the isomorphism (15) from Theorem 3, we obtain (17) and (18). It remains to apply (10) and (11).

Acknowledgements The authors would like to thank the referee for valuable comments which helped to improve the manuscript.

Open Access This article is distributed under the terms of the Creative Commons Attribution 4.0 International License (http://creativecommons.org/licenses/by/4.0/), which permits unrestricted use, distribution, and reproduction in any medium, provided you give appropriate credit to the original author(s) and the source, provide a link to the Creative Commons license, and indicate if changes were made.

\section{References}

1. Almira, J., Luther, U.: Generalized approximation spaces and applications. Math. Nachr. 263-264, 3-35 (2004). https://doi.org/10.1002/mana.200310121

2. Bergh, J., Löfström, J.: Interpolation Spaces. Springer-Verlag, Berlin-Heidelberg-New York (1976)

3. Bieberbach, L.: Analytische fortsetzung. Springer-Verlag, Berlin-Göttingen-Heidelberg (1955)

4. DeVore, R.A.: Nonlinear approximation. Acta Numer. 7(1), 51-150 (1998). https://doi.org/10.1017/ S0962492900002816

5. Dmytryshyn, M., Lopushansky, O.: Operator calculus on the exponential type vectors of operators with point spectrum. In: Book Topology in Banach Spaces, pp. 137-145. Nova, Huntigton, New York (2001)

6. Dmytryshyn, M., Lopushansky, O.: Bernstein-Jackson-type inequalities and Besov spaces associated with unbounded operators. J. Inequal. Appl. 2014, 105 (2014). https://doi.org/10.1186/1029-242X2014-105

7. Giulini, S.: Bernstain and Jackson theorems for the Heisenberg group. J. Aust. Math. Soc. 38, 241-254 (1985). https://doi.org/10.1017/S1446788700023107

8. Giulini, S.: Approximation and Besov spaces on stratified groups. Proc. Am. Math. Soc. 96(4), 569-578 (1986). https://doi.org/10.1090/S0002-9939-1986-0826483-3

9. Gorbachuk, M., Gorbachuk, V.: Approximation of smooth vectors of a closed operator by entire vectors of exponential type. Ukr. Math. J. 47(5), 713-726 (1995). https://doi.org/10.1007/BF01059045

10. Gorbachuk, M.L., Hrushka, YaI, Torba, S.M.: Direct and inverse theorems in the theory of approximation by the Ritz method. Ukr. Math. J. 57(5), 751-764 (2005). https://doi.org/10.1007/s11253-0050225-4

11. Finch, S.R.: Lebesgue Constants. Mathematical Constants. Cambridge University Press, Cambridge (2003)

12. Kato, T.: Perturbation Theory for Linear Operators. Springer-Verlag, Berlin-Heidelberg-New York (1980)

13. Ljubič, J.I., Macaev, V.I.: On operators with separable spectrum. AMS Transl. 47, 89-129 (1965)

14. Nelson, E.: Analytical vectors. Ann. Math. 70(3), 572-615 (1959). https://doi.org/10.2307/1970331

15. Nikolskii, S.: Approximation of Functions of Several Variables and Imbedding Theorems. SpringerVerlag, New York (1975)

16. Peetre, J., Sparr, G.: Interpolation of normed abelian groups. Ann. Mat. Pura ed Appl. 92(1), 217-262 (1972). https://doi.org/10.1007/BF02417949 
17. Pietsch, A.: Approximation spaces. J. Approx. Theory 32(2), 115-134 (1981). https://doi.org/10.1016/ 0021-9045(81)90109-X

18. Triebel, H.: Interpolation Theory. Function Spaces. Differential Operators. North-Holland Publishing Company, Amsterdam-New York-Oxford (1978)

19. Vinogradov, O.L.: Sharp Jackson-type inequalities for approximations of classes of convolutions by entire functions of exponential type. St. Petersburg Math. J. 17(4), 593-633 (2006). https://doi.org/10. 1090/S1061-0022-06-00922-8

Publisher's Note Springer Nature remains neutral with regard to jurisdictional claims in published maps and institutional affiliations. 